\title{
VARIABILIDAD FENOTÍPICA EN ACCESIONES DE Bromus valdivianus Phil.
}

\section{PHENOTYPIC VARIABILITY IN ACCESSIONS OF Bromus valdivianus Phil.}

\author{
José Antonio Blanco ${ }^{1}$, Oscar Balocchi² e Ignacio López ${ }^{2}$ \\ 1 Universidad Católica del Maule, Facultad de Ciencias Agrarias y Forestales, Casilla 7D, Curicó, Chile. 2 Uni- \\ versidad Austral de Chile, Facultad de Ciencias Agrarias, Casilla 567, Valdivia, Chile.e-mail:obalocch@uach.cl
}

\begin{abstract}
Key Words: Bromus valdivianus, native poaceae, phenotypic variability.
\end{abstract}

Bromus valdivianus Phil. is a native poaceae of South America. The objective of this study was to characterize 125 accessions of this species, collected in different areas of the Valdivia province in southern Chile and to analyze the phenotypic variability as a base for future breeding programmes. The accessions were collected from four regions of Valdivia province: NL (north central valley); NP (north foothills); SL (south central valley) and SP (south foothills). After germination in the laboratory the seedlings were transferred to the field. During summer, autumn and winter, 8 qualitative variables and 8 quantitative variables were evaluated. A complete randomized block design with four replicates was used. Variables evaluated in more than one period were subjected to split-plot analysis considering time and space. The data for the multivariate analysis were chosen according to the nature of the interaction between accession and season. When this interaction was significant, the variable was considered over three seasons; conversely, when the interaction was not significant the mean over the seasons was used. A total of 34 variables were obtained and subjected to principal component, cluster and discriminate analysis. Two principal components explained $51 \%$ of the total variation. The cluster analysis distinguished 6 groups. Two groups were clearly distinguished, one formed by accessions with good forage production features and another for its high seed yield. These results are useful for the selection of promissory material, for immediate utilization or for a breeding programme through progenitor selection.

\section{RESUMEN}

Palabras clave: Bromus valdivianus, poaceas nativas, variabilidad fenotípica.

Bromus valdivianus Phil. es una poaceae nativa de América del Sur. El objetivo del estudio fue caracterizar 125 accesiones de esta especie, colectadas en diferentes áreas de la provincia de Valdivia en el sur de Chile y analizar su variabilidad fenotípica como base para un futuro programa de mejoramiento. Las accesiones fueron colectadas de cuatro áreas: NL (valle central norte); NP (precordillera andina norte); SL (valle central sur) y SP (precordillera andina sur). Ocho variables cuantitativas y ocho variables cualitativas fueron evaluadas durante el verano, otoño e invierno. Se utilizó un diseño experimental de bloques completos al azar, con cuatro repeticiones. Las variables evaluadas en más de un periodo fueron sometidas a un análisis de parcela dividida. Los datos incorporados en el análisis multivariado fueron elegidos de acuerdo a la naturaleza de la interacción accesión por periodo. Cuando la interacción fue significativa las variables fueron consideradas independientemente en los tres periodos; cuando no fue significativa se usó como variable el promedio de los periodos. Un total de 34 variables que fueron sometidas a análisis de componentes principales, agrupamiento y análisis discriminante. Los dos primeros componentes principales explicaron el $51 \%$ de la variación total. En el análisis de agrupamiento se distinguieron seis grupos. Dos grupos contrastantes, uno formado por accesiones con características asociadas a una buena producción de forraje y otro asociado a características de mayor producción de semillas. Estos resultados son útiles en la selección de material promisorio de utilización inmediata o para uso como progenitores en programas de mejoramiento. 


\section{INTRODUCCIÓN}

El género Bromus contiene aproximadamente 400 especies con una amplia distribución geográfica y gran diversidad de hábitats (Pillay, 1995). Se encuentra en climas templados o tropicales del Hemisferio Norte, América del Sur y en la parte sur de África. Algunas especies originarias de zonas climáticas con inviernos templados tienen un patrón de crecimiento estacional apropiado y son usadas como planta forrajera en países con clima templado (Stewart, 1996) Las especies más conocidas de este género y usadas comercialmente en Australia y Nueva Zelandia son $B$. willdenowii y B. stamineus.

Bromus valdivianus Phil., es una especie hexaploide de autopolinización (Sutherland, 1994), perteneciente a la sección Ceratochloa y nativa de América del Sur. Su potencial forrajero no ha sido explotado, crece actualmente como una planta silvestre y es una especie promisoria por su resistencia, rusticidad y facilidad de manejos (Balocchi y López, 2001). Por lo tanto, esta especie presenta un gran potencial para trabajos de selección y mejoramiento como especie forrajera adaptada a las condiciones edafoclimáticas de la zona sur de Chile.

Bromus valdivianus es una especie perenne, de 0,4 a 0,7 m de altura con vainas de color ocre cerradas y pilosas. Láminas foliares planas, de 10 a $15 \mathrm{~cm}$ de largo por 2 a $4 \mathrm{~mm}$ de ancho, cubiertas de pelos largos poco densos y márgenes dentados. Lígula membranosa, de 1 a $3 \mathrm{~mm}$ no decurrente, de borde obtuso y dentado. Panoja algo laxa, de 5 a $10 \mathrm{~cm}$ de largo; espiguillas de 20 a $22 \mathrm{~mm}$ de largo incluyendo las aristas y antecios pubescentes. El fruto es un cariopsis linear, algo comprimido, surcado, tiene un ápice con pelos blancos, de color café y mide aproximadamente $6,5 \mathrm{~mm}$ de largo. Posee una arista que suele medir de 3 a 5,5 mm de largo (Muñoz, 1980).

Stewart (1996) estudió, en Nueva Zelandia, el rol potencial de diez especies de Bromus de la sección Ceratochloa, indicando que son plantas de baja tolerancia a suelos con mal drenaje o de textura pesada, muy ácidos, o con altos niveles de aluminio. Presentan enraizamiento moderadamente profundo con buena tolerancia a sequía. El forraje producido es generalmente de alta calidad y palatabilidad. En el sur de Chile,
Balocchi y López (2001), estudiando las especies componentes de las praderas, encontraron que $B$. valdivianus se destacó por su alto rendimiento de forraje y calidad nutritiva, mostrando un comportamiento superior a especies exóticas naturalizadas como Dactylis glomerata.

Bromus valdivianus domina una diversidad de condiciones ambientales en la Provincia de Valdivia, Chile (López et al., 1997). La capacidad de las plantas a ajustarse y sobrevivir en ambientes diversos se basa en la variabilidad genética dada por la generación de ecotipos o a través de la plasticidad fenotípica (Vanhala et al., 2004).

Considerando las características favorables de esta especie para la alimentación de herbívoros y su condición de planta nativa de la región, el presente estudio tuvo como objetivo determinar la variabilidad fenotípica de accesiones de $B$. valdivianus colectadas en la Provincia de Valdivia, Chile.

\section{MATERIAL Y MÉTODO}

\section{Lugar del estudio}

El estudio se desarrolló en primera instancia, en el Laboratorio de Forrajeras del Instituto de Producción Animal, de la Universidad Austral de Chile, donde se germinaron las semillas de las accesiones utilizando macetas con $100 \mathrm{~g}$ de suelo, con el propósito de lograr un desarrollo adecuado de las plántulas y asegurar de esta manera el posterior trasplante a terreno.

El período de evaluación del ensayo abarcó el verano, otoño e invierno. Se utilizó un diseño de bloques completos al azar (Steel y Torrie, 1988), con cuatro repeticiones y 125 tratamientos (las accesiones de B. valdivianus). Se plantaron dos plantas de la misma accesión por parcela, con una separación entre plantas de $0,4 \mathrm{~m}$ y entre accesiones de $0,5 \mathrm{~m}$. Las variables analizadas en el experimento se muestran en el Cuadro 1. El ensayo se mantuvo libre de malezas mediante control químico en una primera instancia y posteriormente control mecánico y manual. $\mathrm{Al}$ establecimiento se fertilizó con $45 \mathrm{~kg}$ de $\mathrm{N} \mathrm{ha}^{-1}$, $70 \mathrm{~kg}$ de $\mathrm{P}_{2} \mathrm{O}_{5}$ ha- $^{-1}$ y $60 \mathrm{~kg}$ de $\mathrm{K}_{2} \mathrm{O}$ ha- ${ }^{-}$.

\section{Material vegetal}

Se evaluaron 125 accesiones de B. valdivianus, 
Cuadro 1. Variables evaluadas en el estudio

Table 1.Description of the variables analyzed during the study

\begin{tabular}{lcl}
\hline Variables & Abreviación $^{\mathrm{I}}$ & \multicolumn{1}{c}{ Unidad } \\
\hline CUALITATIVAS & & \\
Adaptación & ADAP & 1 (malo) -5 (excelente) \\
Daño por enfermedades & ENF & 1 (presencia) -5 (severo) \\
Hábito de crecimiento & HAB & 1 (erecto) -5 (postrado) \\
Daño por helada & HEL & 1 (ausencia) -5 (grave) \\
Inflorescencias & INF & 1 (escasa) -5 (abundante) \\
Daño por insectos & INS & 1 (presencia) - 5 (grave) \\
Rebrote & REB & 1 (bajo) - 5 (alto) \\
Vigor de planta & VIG & 1 (débil) - 5 (vigoroso) \\
\hline CUALITATIVAS & & \\
\hline Altura de planta & ALT & $\mathrm{cm}$ \\
Ancho de lámina & ANCH & $\mathrm{cm}$ \\
Porcentaje de cobertura & COB & $\%$ \\
Diámetro base de la planta & DIAM & $\mathrm{cm}$ \\
Porcentaje de hoja & HOJ & $\%$ \\
Largo de lámina & LARG & $\mathrm{cm}$ \\
Materia seca & MS & g planta \\
Producción de semilla & SEM & g planta \\
& & \\
\hline
\end{tabular}

1: Las variables medidas en Otoño, Invierno y Verano se identifican con la letra $\mathrm{o}, \mathrm{i}, \mathrm{v}$, respectivamente

colectadas en la Provincia de Valdivia, Chile. Con el objetivo de obtener una colección representativa, en base a estudios realizados por Montaldo y Fuentes (1981) y Montaldo et al. (1982), considerando principalmente las características edafoclimáticas predominantes, se dividió la Región en cuatro zonas: Valle central norte (NL); Precordillera andina norte (NP); Valle central sur (SL) y Precordillera andina sur (SP). Cada accesión correspondió a semilla proveniente de una sola planta. Toda la información se adecuó a los descriptores propuestos por International Board for Plant Genetic Resources, IBPGR (Tyler et al., 1985); el Centro Internacional de Agricultura Tropical, CIAT (Toledo y Schultze-Kraft, 1982), y la Empresa Brasileira de Pesquisa Agropecuaria, EMBRAPA (Comastri y Pott, 1982).

Diseño experimental y análisis estadístico Se utilizó un diseño de bloques completos al azar, con cuatro repeticiones y 125 tratamientos (las accesiones de $B$. valdivianus). La unidad experimental estuvo representada por parcelas cada una con dos plantas separadas a $0,4 \mathrm{~m}$ y entre accesiones de $0,5 \mathrm{~m}$ Las variables analizadas en el experimento se muestran en el Cuadro 1.

Se efectuó un análisis de varianza para cada una de las variables en cada época (estación). Para aquellas variables medidas en más de una época se efectuó el análisis conjunto utilizando el análisis de parcelas divididas en el tiempo y el espacio (Steel y Torrie, 1988).

En el análisis univariado se determinó la naturaleza de la interacción accesión por época. Si esta interacción fue significativa y más importante que el efecto principal de la accesión para una variable particular, se consideró esta variable medida en las tres épocas como variables diferentes en el análisis multivariado. Contrariamente, si los efectos principales de la accesión fueron más importantes que la interacción accesión por época, se utilizó la media para cada accesión sobre bloques y épocas en el análisis multivariado. Para realizar esta determinación se utilizó la siguiente relación para el valor de F, $\mathrm{F}=$ (cuadrado medio de la accesión)/(cuadrado medio de la interacción accesión por época por bloque). Si esta $\mathrm{F}$ fue significativa, se utilizó la media sobre épocas y bloques. Si la F no fue significativa se utilizó la media sobre bloques y se empleó las épocas como diferentes variables.

De acuerdo con el procedimiento descrito se obtuvieron 34 variables que junto a las 125 ac- 
Cuadro 2. Cuadrados medios del análisis conjunto, de las variables que se repiten en más de una época. Table 2. Mean squares of joint analysis of the variables which are repeated in more than one season.

\begin{tabular}{|c|c|c|c|c|c|c|c|c|c|c|c|c|c|c|c|c|c|c|c|c|c|c|c|c|}
\hline \multirow{3}{*}{$\begin{array}{l}\text { Causa de } \\
\text { variación } \\
\text { Repetición (R) }\end{array}$} & \multirow{3}{*}{$\frac{\text { g.l. }}{3}$} & \multicolumn{23}{|c|}{ Variables evaluadas } \\
\hline & & ENF & \multicolumn{2}{|c|}{ HAB } & \multicolumn{2}{|c|}{$\mathrm{INF}^{2}$} & \multicolumn{2}{|c|}{ INS } & \multicolumn{2}{|c|}{ REB } & \multicolumn{2}{|c|}{ VIG } & \multicolumn{2}{|c|}{ ALT } & \multicolumn{2}{|c|}{$\mathrm{ANCH}$} & \multicolumn{2}{|l|}{$\mathrm{COB}$} & \multicolumn{2}{|c|}{ DIAM } & \multicolumn{2}{|l|}{ LARG } & \multicolumn{2}{|l|}{ MS } \\
\hline & & 2.21 & 0.42 & & 1.53 & & 0.31 & & 18.08 & & 10.25 & & 1488.95 & & 0.08 & & 2780.88 & & 993.97 & & 200.78 & & 3807.48 & \\
\hline Epoca $(E)$ & 2 & $1.71 \mathrm{~ns}$ & 10.25 & $*$ & 297.78 & $* *$ & 0.68 & ns & 270.71 & $* *$ & 192.37 & $* *$ & 7924.14 & $* *$ & 3.93 & $* *$ & 4533.03 & ns & 853.57 & $* *$ & 25244.86 & $* *$ & 83101.54 & * \\
\hline $\mathrm{R} \times \mathrm{E}$ & 6 & 0.48 & 1.22 & & 0.98 & & 1.27 & & 3.47 & & 0.96 & & 115.14 & & 0.06 & & 1785.38 & & 10.85 & & 261.06 & & 517.42 & \\
\hline Ecotipo (Ec) & 124 & $0.13 * *$ & 2.37 & $* *$ & 1.36 & $* *$ & 0.11 & $*$ & 1.77 & $* *$ & 1.74 & $* *$ & 131.70 & ** & 0.06 & $* *$ & 556.38 & $*$ & 8.66 & $* *$ & 66.90 & $* *$ & 913.02 & ** \\
\hline $\mathrm{R} \times \mathrm{Ec}$ & 372 & $0.06 \mathrm{~ns}$ & 0.24 & $*$ & 0.13 & $* *$ & 0.09 & ns & 0.57 & $* *$ & 0.68 & $* *$ & 21.99 & $* *$ & 0.02 & ns & 155.17 & $\begin{array}{l}* \\
*\end{array}$ & 4.12 & $* *$ & 25.59 & $* *$ & 112.33 & ** \\
\hline $\mathrm{E} \times \mathrm{Ec}$ & 248 & $0.10 * *$ & 2.01 & $* *$ & 0.85 & $* *$ & 0.08 & $\mathrm{~ns}$ & 0.44 & ** & 0.21 & $* *$ & 26.23 & $* *$ & 0.03 & ** & 79.71 & $\begin{array}{l}* \\
*\end{array}$ & 0.56 & ns & 27.39 & $* *$ & 225.16 & ** \\
\hline Error & 744 & 0.06 & 0.20 & & 0.10 & & 0.09 & & 0.27 & & 0.13 & & 8.30 & & 0.01 & & 34.34 & & 0.52 & & 18.27 & & 54.30 & \\
\hline Total & 1499 & & & & & & & & & & & & & & & & & & & & & & & \\
\hline Media & & 1.1 & 2.8 & & 1.6 & & 1.1 & & 4.0 & & 4.0 & & 25.0 & & 0.8 & & 66.4 & & 10.5 & & 26.0 & & 30.5 & \\
\hline $\mathrm{CV}, \%$ & & 23.5 & 16.3 & & 19.62 & & 27.3 & & 13.2 & & 9.0 & & 11.5 & & 16.9 & & 8.8 & & 6.9 & & 16.5 & & 24.1 & \\
\hline
\end{tabular}

2, Basado en dos épocas, primavera y verano.

Nivel de significancia: $* \mathrm{p}<0,05 ; * * \mathrm{p}<0,01 ; \mathrm{ns}=$ no significativo.

cesiones de $B$. valdivianus, permitió construir una matriz de 125 x 34 , los valores fueron estandarizados con media cero y varianza uno y sujetos a los análisis de componente principal, conglomerados por el método k-means, y discriminante (Hair et al., 1992; Härdle y Simar, 2007).

\section{RESULTADOS Y DISCUSIÓN}

Los resultados del análisis conjunto de las variables medidas en más de una época se indican en el Cuadro 2. La interacción época x accesión no fue significativa para las variables daño por insectos (INS) y el diámetro de la base de la planta (DIAM). Para el efecto principal de accesión, INS fue significativo $(\mathrm{p}<0,05)$ y DIAM altamente significativo $(p<0,01)$; consecuentemente se obtuvo el promedio de cada accesión a través de épocas y bloques. El resto de las variables presentan interacción significativa; por tanto, se utilizaron las variables medidas en las tres épocas como diferentes para el análisis multivariado.

El grado de adaptación (ADAP), daño por heladas (HEL), cantidad de inflorescencia (INF), porcentaje de hoja (HOJ) y producción de semilla (SEM) se evaluaron en una sola época y se ingresaron directamente al análisis multivariado. Se obtuvieron en total 34 variables, las cuales se presentan en el Cuadro 3, indicando sus medidas de tendencia central y dispersión.

\section{Análisis de componentes principales}

Los coeficientes de correlación entre las 34 variables originales y los dos primeros componentes explican un $51 \%$ de la variación total (Figura 1). El primer componente principal (CP1), explica el 39,4\% de la variación total y se encuentra asociado con las variables, porcentaje de cobertura, vigor de planta, materia seca, altura de planta, grado de adaptación, diámetro de la base de la planta, rebrote de planta, porcentaje de hoja y el largo de lámina; se asociaron positivamente a la formación de este componente. En contraste a éstas estuvo el hábito de crecimiento, especialmente en el otoño e invierno. Las variables ancho de lámina, susceptibilidad al daño por enfermedades, producción de semilla, inflorescencia, susceptibilidad al daño por insectos y daño por helada aportaron muy poco en explicar las diferencias entre accesiones. Dado que las variables asociadas al primer componente indicaron una buena capacidad de las accesiones de cubrir el suelo, una mayor producción de biomasa, además de un buen vigor y adaptación, este componente estaría discriminando aquellas accesiones con mayor producción de forraje, muy especialmente en otoño e invierno.

En el segundo componente, las mayores contribuciones estuvieron dadas por la altura de planta en verano, producción de semilla, inflorescencia y el ancho de lámina en verano, las que se asociaron positivamente. Por el contrario se asoció negativamente el hábito de crecimiento en verano y porcentaje de hoja. Este componente explicó el 12,1\% de la variación total y discriminó las accesiones en cuanto a producción de semillas, siendo mayor ésta en las accesiones 


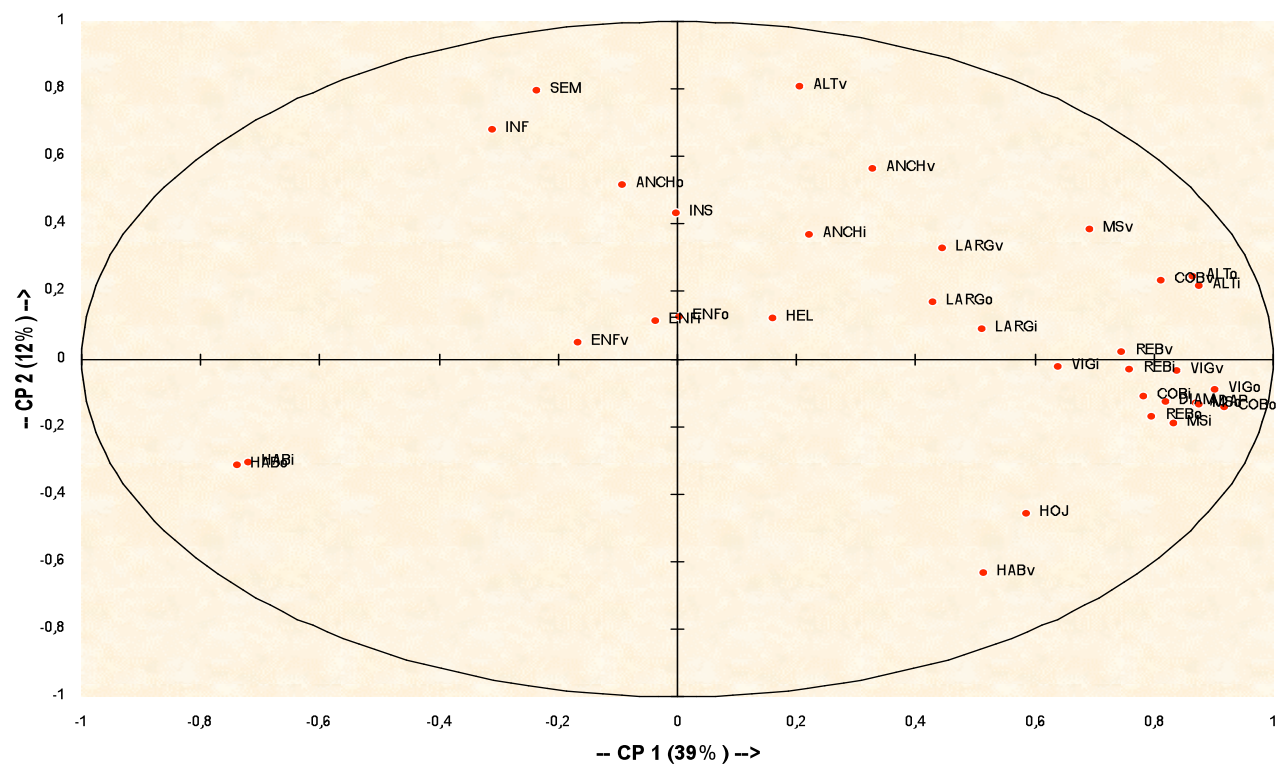

Figura 1. Círculo de correlación de las variables entre el primer y segundo componente.

Figure 1. Correlation circle of the variables between the first and the second component

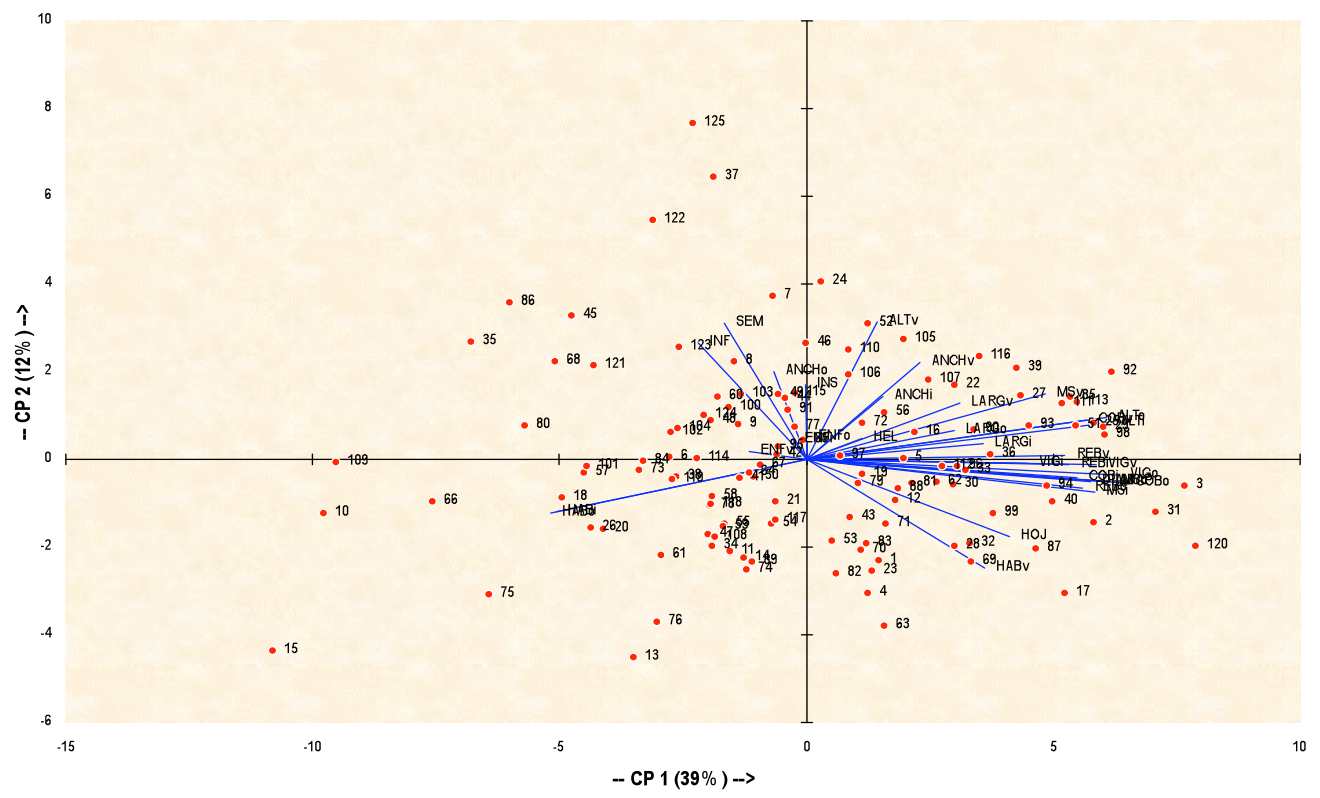

Figura 2. Biplano de los 34 caracteres agronómicos con los 125 ecotipos de Bromus valdivianus. Figure 2. Biplane of the 34 agronomic characters with the 125 ecotypes of Bromus valdivianus. 
de crecimiento erecto y de menor porcentaje de hojas.

La Figura 1 muestra los dos primeros componentes y se observa la asociación entre las variables. Las variables relacionadas a factores de producción de forraje se asociaron al primer componente: cobertura de otoño e invierno, vigor de planta, materia seca en otoño e invierno, adaptación, diámetro y rebrote. Las variables altura de planta en invierno y otoño y la cobertura de verano se asociaron entre ellas y al primer componente. Las variables relacionadas a la producción de semilla se asociaron al segundo componente. $\mathrm{Al}$ ordenar las accesiones en base a los valores de los componentes principales se observó la asociación de ellas en relación a las variables analizadas y permitió identificar a las accesiones con mayor aptitud forrajera. Esta técnica proporcionó una interpretación biológica (Franco et al., 1997) y fenotípica (Smith et al., 1995), del material en estudio. Para el primer componente, que correspondió a la habilidad de producción de forraje, se destacaron las accesiones $120 ; 3$ y 31 , con mayor cobertura, vigor, materia seca, altura, adaptación y diámetro de planta. Caracteres sobresalientes y deseables

Cuadro 3. Parámetros estadísticos de tendencia central y dispersión para las variables analizadas en las tres épocas.

Table 3. Statistical parameters of central tendency and dispersion for the analyzed variables in the three seasons

\begin{tabular}{|c|c|c|c|c|}
\hline Variables & $\begin{array}{l}\text { Rango de } \\
\text { Variación }\end{array}$ & Promedio & $\begin{array}{c}\text { Desviación } \\
\text { estándar }\end{array}$ & $\begin{array}{c}\text { Coeficiente } \\
\text { variación } \\
(\%)\end{array}$ \\
\hline \multicolumn{5}{|l|}{ Cualitativas: } \\
\hline Adaptación & $2,0-5,0$ & 3,9 & 0,6 & 16,4 \\
\hline Daño por enfermedad verano & $1,0-3,0$ & 1,1 & 0,3 & 27,7 \\
\hline Daño por enfermedad otoño & $1,0-3,0$ & 1,1 & 0,3 & 26,6 \\
\hline Daño por enfermedad invierno & $1,0-2,0$ & 1,0 & 0,1 & 10,7 \\
\hline Hábito de crecimiento verano & $1,0-5,0$ & 2,7 & 0,5 & 18,6 \\
\hline Hábito de crecimiento otoño & $2,0-5,0$ & 3,0 & 0,4 & 14,3 \\
\hline Hábito de crecimiento invierno & $1,0-5,0$ & 2,7 & 0,5 & 17,6 \\
\hline Daño por helada & $1,0-3,0$ & 1,9 & 0,5 & 24,1 \\
\hline Inflorescencia & $1,0-5,0$ & 2,2 & 0,4 & 19,9 \\
\hline Daño por insectos & $1,0-3,0$ & 1,1 & 0,3 & 27,3 \\
\hline Rebrote de verano & $2,0-5,0$ & 3,2 & 0,7 & 22,1 \\
\hline Rebrote de otoño & $2,0-5,0$ & 4,2 & 0,6 & 13,7 \\
\hline Rebrote de invierno & $3,0-5,0$ & 4,6 & 0,5 & 11,9 \\
\hline Vigor de planta verano & $2,0-5,0$ & 3,9 & 0,6 & 14,4 \\
\hline Vigor de planta otoño & $2,0-5,0$ & 3,5 & 0,6 & 17,1 \\
\hline Vigor de planta invierno & $3,0-5,0$ & 4,7 & 0,5 & 11,1 \\
\hline \multicolumn{5}{|l|}{ Cuantitativas: } \\
\hline Altura de planta verano & $9,0-51,0$ & 21,8 & 3,7 & 17,0 \\
\hline Altura de planta otoño & $12,5-49,0$ & 29,4 & 3,9 & 13,3 \\
\hline Altura de planta invierno & $11,0-36,5$ & 23,7 & 3,1 & 13,0 \\
\hline Ancho de lámina verano & $0,2-1,5$ & 0,7 & 0,1 & 18,1 \\
\hline Ancho de lámina otoño & $0,4-1,7$ & 0,9 & 0,2 & 18,0 \\
\hline Ancho de lámina invierno & $0,5-1,3$ & 0,8 & 0,1 & 15,6 \\
\hline Porcentaje de cobertura verano & $30,0-98,0$ & 65,2 & 9,0 & 13,8 \\
\hline Porcentaje de cobertura otoño & $35,0-98,0$ & 69,8 & 9,4 & 13,5 \\
\hline Porcentaje de cobertura invierno & $35,0-85,0$ & 64,1 & 7,3 & 11,4 \\
\hline Diámetro base de la planta & $5,3-17,0$ & 10,5 & 0,7 & 6,9 \\
\hline Porcentaje de hoja & $28,8-100,0$ & 72,7 & 12,4 & 17,1 \\
\hline Largo de lámina verano & $8,0-36,0$ & 19,6 & 2,9 & 14,7 \\
\hline Largo de lámina otoño & $17,5-59,0$ & 33,7 & 5,9 & 17,7 \\
\hline Largo de lámina invierno & $13,0-45,0$ & 24,6 & 4,3 & 17,5 \\
\hline Materia seca verano & $2,9-109,2$ & 40,5 & 10,8 & 26,7 \\
\hline Materia seca otoño & $8,5-86,6$ & 35,1 & 9,3 & 26,6 \\
\hline Materia seca invierno & $4,0-42,0$ & 16,0 & 4,1 & 25,8 \\
\hline Producción de semilla & $1,0-82,9$ & 22,0 & 6,5 & 29,3 \\
\hline
\end{tabular}


Cuadro 4. Valores medios de las 34 variables utilizadas en el análisis de agrupamiento, valor $\mathbf{F}$ y su probabilidad.

Table 4. Mean values of the 34 variables used in the cluster analysis, $F$ value and its probability.

\begin{tabular}{|c|c|c|c|c|c|c|c|c|}
\hline \multirow[b]{2}{*}{ Variable } & \multicolumn{7}{|c|}{ Grupo $^{1}$} & \multirow[b]{2}{*}{ Valor $\mathbf{P}^{3}$} \\
\hline & $1(34)$ & $2(35)$ & $3(15)$ & $4(31)$ & $5(9)$ & $6(1)^{2}$ & Valor F & \\
\hline Adaptación & 3,8 & 4,3 & 4,4 & 3,7 & 3,0 & 3,3 & 27,494 & 0,001 \\
\hline Daño por enfermedad verano & 1,2 & 1,1 & 1,0 & 1,1 & 1,1 & 1,0 & 1,005 & 0,418 \\
\hline Daño por enfermedad otoño & 1,1 & 1,1 & 1,2 & 1,2 & 1,1 & 1,0 & 1,596 & 0,166 \\
\hline Daño por enfermedad invierno & 1,0 & 1,0 & 1,0 & 1,0 & 1,1 & 1,0 & 1,647 & 0,153 \\
\hline Hábito de crecimiento verano & 2,7 & 3,2 & 3,6 & 2,0 & 2,2 & 1,0 & 16,685 & 0,001 \\
\hline Hábito de crecimiento otoño & 3,2 & 2,8 & 2,3 & 3,1 & 3,6 & 3,0 & 19,792 & 0,001 \\
\hline Hábito de crecimiento invierno & 2,9 & 2,5 & 2,0 & 2,8 & 3,8 & 2,8 & 13,698 & 0,001 \\
\hline Daño por helada & 2,0 & 1,9 & 1,9 & 1,9 & 1,7 & 2,3 & 0,813 & 0,543 \\
\hline Inflorescencias & 1,9 & 2,0 & 1,5 & 2,8 & 2,2 & 4,8 & 21,595 & 0,001 \\
\hline Daño por insectos & 1,1 & 1,1 & 1,1 & 1,1 & 1,1 & 1,3 & 0,943 & 0,456 \\
\hline Rebrote de verano & 2,9 & 3,5 & 4,0 & 2,9 & 2,7 & 2,8 & 23,427 & 0,001 \\
\hline Rebrote de otoño & 4,2 & 4,5 & 4,6 & 4,2 & 3,3 & 4,0 & 23,946 & 0,001 \\
\hline Rebrote de invierno & 4,5 & 4,7 & 4,9 & 4,6 & 3,8 & 4,5 & 26,529 & 0,001 \\
\hline Vigor de planta verano & 3,8 & 4,0 & 4,3 & 3,8 & 3,2 & 3,8 & 20,692 & 0,001 \\
\hline Vigor de planta otoño & 3,3 & 3,8 & 4,2 & 3,2 & 2,6 & 3,0 & 35,134 & 0,001 \\
\hline Vigor de planta invierno & 4,7 & 4,8 & 4,9 & 4,7 & 4,2 & 4,8 & 8,705 & 0,001 \\
\hline Altura de planta verano & 20,0 & 21,1 & 23,5 & 24,1 & 18,7 & 35,0 & 11,795 & 0,001 \\
\hline Altura de planta otoño & 27,4 & 31,4 & 35,4 & 28,7 & 21,9 & 28,3 & 28,434 & 0,001 \\
\hline Altura de planta invierno & 22,3 & 25,7 & 27,8 & 23,0 & 17,4 & 23,1 & 30,653 & 0,001 \\
\hline Ancho de lámina verano & 0,6 & 0,7 & 0,8 & 0,7 & 0,6 & 1,0 & 8,370 & 0,001 \\
\hline Ancho de lámina otoño & 0,9 & 0,9 & 0,8 & 0,9 & 0,9 & 1,0 & 1,261 & 0,285 \\
\hline Ancho de lámina invierno & 0,8 & 0,8 & 0,8 & 0,8 & 0,8 & 0,9 & 0,638 & 0,671 \\
\hline Porcentaje de cobertura verano & 57,3 & 69,6 & 80,1 & 65,5 & 52,1 & 72,5 & 64,880 & 0,001 \\
\hline Porcentaje de cobertura otoño & 66,2 & 75,0 & 81,1 & 66,8 & 55,3 & 62,5 & 61,232 & 0,001 \\
\hline Porcentaje de cobertura invierno & 62,4 & 67,6 & 68,0 & 63,5 & 52,5 & 58,8 & 23,823 & 0,001 \\
\hline Diámetro base de la planta & 10,1 & 11,1 & 11,7 & 10,2 & 9,2 & 10,4 & 42,999 & 0,001 \\
\hline Porcentaje de hoja & 75,3 & 78,7 & 88,9 & 60,5 & 58,5 & 38,1 & 44,933 & 0,001 \\
\hline Largo de lámina verano & 18,4 & 20,5 & 22,3 & 18,9 & 18,5 & 22,6 & 6,350 & 0,001 \\
\hline Largo de lámina otoño & 32,3 & 35,5 & 35,7 & 32,6 & 32,3 & 30,5 & 4,865 & 0,001 \\
\hline Largo de lámina invierno & 24,2 & 25,7 & 26,4 & 23,8 & 22,5 & 21,8 & 4,907 & 0,001 \\
\hline Materia seca verano & 28,7 & 45,1 & 63,5 & 41,2 & 23,1 & 73,7 & 60,890 & 0,001 \\
\hline Materia seca otoño & 29,8 & 41,9 & 54,4 & 29,1 & 18,0 & 24,8 & 67,952 & 0,001 \\
\hline Materia seca invierno & 14,2 & 18,9 & 20,5 & 14,5 & 9,6 & 10,6 & 33,950 & 0,001 \\
\hline Producción de semilla & 17,2 & 20,2 & 12,8 & 32,7 & 21,2 & 67,3 & 26,065 & 0,001 \\
\hline
\end{tabular}

${ }^{1}$ Números entre paréntesis indican el número de ecotipos en cada grupo.

${ }^{2}$ Grupo 6 corresponde al ecotipo 125

${ }^{3} \mathrm{P}=$ Probabilidad que la variable no difiere significativamente entre grupos.

en un ecotipo para la producción de forraje y esta situación se presentó en las tres épocas. En cambio, las accesiones $15 ; 10$ y 109 reunieron las características menos deseables en estos caracteres (Figura 2).

El segundo componente destacó las características reproductivas. El aumento positivo de los valores de las accesiones en este componente indicó un incremento en la producción de semilla. Se destacaron las accesiones 125; 37 y 122, cuyo origen fue la región NL, con un rendimiento de semilla de 67,$3 ; 43,4$ y 44,7 gp planta ${ }^{-1}$, respectivamente. Como expresión de los procesos reproductivos la accesión 125 fue la planta con mayor altura $(35,0 \mathrm{~cm})$, mientras las menores alturas las presentaron las accesiones $13 ; 15$ y 63 con 12,4; 15,5 y 13,8 cm con producciones de semilla de 9,$8 ; 16,4$ y 9,3 gp planta ${ }^{-1}$, respectivamente (Figura 2).

En la Figura 2, la dirección de los vectores indica el grado de asociación de las variables con las accesiones y la longitud del vector determina el poder discriminante de la asociación (Derksen et al., 1993 y Hazen et al., 1997 ). En base a la dirección del vector, se observa que existe un alto grado de asociación entre las variables y las accesiones. Con relación a la longitud de los vectores, gran parte de las variables son buenas discriminadoras. Los menos discriminantes fueron daño -patológico y 
entomológico obtenido en las tres épocas y daño por heladas, ya que presentan una menor longitud del vector y presentaron una calificación baja en las diferentes evaluaciones. Como estos vectores muestran la variabilidad fenotípica, las variables con menor longitud no logran discriminar a las accesiones por dichas características. Este biplano permite destacar que las accesiones con mayor producción de semillas están asociadas a variables que representan un mayor número de inflorescencias, menor porcentaje de hojas y un hábito de crecimiento más erecto. Las accesiones con mayor porcentaje de hojas están asociadas a un hábito de crecimiento postrado. Cabe destacar que accesiones con hábito de crecimiento postrado en otoño e invierno son menos productores de forraje. En general, todas las variables de importancia agronómica relacionadas con la producción de forraje se encuentran a la derecha de la Figura 2 y las accesiones que presentan estas características se encuentran alrededor de las mismas.

\section{Análisis de agrupamiento}

El análisis de agrupamiento se realizó por la técnica K-media (K-means), en base a la matriz básica de datos, con datos estandarizados a media cero y varianza uno. Se eligieron seis grupos, ya que de este modo se obtuvo una agrupación más homogénea de las accesiones, basándose en caracteres de interés agronómico. La formación de los grupos no estuvo relacionada con la procedencia de las accesiones, encontrándose accesiones de distinta procedencia en todos los grupos. Existieron diferencias significativas entre grupos para la mayoría de las variables analizadas, excepto para el daño por enfermedades en las tres épocas, daño por heladas e insectos y el ancho de lámina en otoño e invierno (Cuadro 4). Uno de los grupos quedó constituido por una sola accesión, la número 125 , que se destaca por su mayor producción de semilla y altura de planta. En el Cuadro 4 se muestra el número de accesiones que conforman cada grupo: $34 ; 35$; $15 ; 31 ; 9$ y 1 accesiones para el primer al sexto grupo respectivamente. Las accesiones con las mejores características agronómicas de interés forrajero, ya sea para utilización inmediata, asî como para un programa de mejoramiento se encuentran incluidas en el Grupo 3. Este grupo presentó en promedio la mayor producción de materia seca en las tres épocas de evaluación. En la época de verano presentó un rendimiento de 63,5 $\pm 8,64$ gp planta $^{-1}$ con un coeficiente de variación de 13,6\%. El rango de variación entre la accesión con menor y mayor rendimiento fue de 47,3 a 86,3 gp planta $^{-1}$ que correspondieron a las accesiones 31 y 98 , respectivamente. En otoño el promedio fue de 54,4 $\pm 6,37 \mathrm{gp} \mathrm{planta}^{-1}$ con un coeficiente de variación relativamente bajo $(11,7 \%)$ y un rango de variación de 43,4 a 64,8 gp planta ${ }^{-1}$ dados por las accesiones 111 y 120 , respectivamente. Finalmente, para el período invernal el promedio de producción fue de 20,5 $\pm 2,73 \mathrm{gp} \mathrm{planta}^{-1}$ con un coeficiente de variación de 13,3\%. El rango varió entre 14,5 gp planta ${ }^{-1}$ para la accesión menos productiva y $24,5 \mathrm{~g} /$ planta para la más productiva, correspondiente a las accesiones 92 y 98, respectivamente.

El Grupo 4 incorporó las accesiones que poseían una buena producción de semilla, con un promedio de $32.7 \pm 8,04$ gp planta $^{-1}$ y con un coeficiente de variación de $24,6 \%$. El rango de variación fue de 19,3 a 46,9 gp planta ${ }^{-1}$, correspondiendo a las accesiones 48 y 86 , respectivamente. El número de inflorescencias por planta se relacionó estrechamente a producción de semillas con una calificación promedio de 2,9 $\pm 0,60$, con un coeficiente de variación relativamente alto $(21,1 \%)$ y un rango de variación de 1,7 a 4,0 que correspondió a las accesiones 50 y 7 , respectivamente.

El Grupo 5 tiene características contrarias al Grupo 3 y reúne las accesiones con menor producción de forraje. En verano obtuvo una producción promedio de $23,1 \pm 7,88$ gp planta $^{-1}$; con un coeficiente de variación de $34,1 \%$ y un rango de variación de 12,5 a 39,2 gp planta ${ }^{-1}$, la menor producción la presentó la accesión 101 y la mayor del grupo la accesión 80. En otoño obtuvo un promedio de 18,0 $\pm 3,36$ gp planta $^{-1}$; con un coeficiente de variación de $18,7 \%$ y un rango de variación de 12,3 gp planta-1 (accesión $^{-1}$ 119) a 25,0 gp planta-1 (accesión 20). En invierno el promedio de producción fue de 9,6 4 1,18 gp planta-1; con un coeficiente de variación de 12,3\%; la accesión 15 fue la de menor producción con 8,2 gp planta-1 y la accesión 80 tiene la producción más alta del grupo (11,3 gp planta $\left.^{-1}\right)$. Este grupo se caracterizó por contar con 
Cuadro 5. Coeficiente de correlación entre las variables originales discriminantes y las cuatro primeras funciones discriminantes.

Table 5. Correlation coefficient amongst the original discriminate variables and the four first discriminate functions.

\begin{tabular}{|c|c|c|}
\hline \multirow[b]{2}{*}{ Variables originales } & \multicolumn{2}{|c|}{ Funciones discriminantes } \\
\hline & 1 & 2 \\
\hline Adaptación & 0,71 & $-0,03$ \\
\hline Daño por enfermedad verano & $-0,18$ & 0,00 \\
\hline Daño por enfermedad otoño & 0,04 & 0,19 \\
\hline Daño por enfermedad invierno & $-0,04$ & $-0,01$ \\
\hline Hábito de crecimiento verano & 0,54 & $-0,41$ \\
\hline Hábito de crecimiento otoño & $-0,70$ & $-0,11$ \\
\hline Hábito de crecimiento invierno & $-0,62$ & $-0,05$ \\
\hline Daño por helada & 0,07 & $-0,04$ \\
\hline Inflorescencias & $-0,37$ & 0,65 \\
\hline Daño por insectos & 0,05 & 0,17 \\
\hline Rebrote de verano & 0,71 & $-0,11$ \\
\hline Rebrote de otoño & 0,64 & 0,03 \\
\hline Rebrote de invierno & 0,63 & 0,12 \\
\hline Vigor de planta verano & 0,69 & 0,01 \\
\hline Vigor de planta otoño & 0,79 & $-0,07$ \\
\hline Vigor de planta invierno & 0,45 & 0,06 \\
\hline Altura de planta verano & 0,21 & 0,55 \\
\hline Altura de planta otoño & 0,76 & 0,13 \\
\hline Altura de planta invierno & 0,76 & 0,09 \\
\hline Ancho de lámina verano & 0,38 & 0,38 \\
\hline Ancho de lámina otoño & $-0,13$ & 0,12 \\
\hline Ancho de lámina invierno & 0,11 & 0,00 \\
\hline Porcentaje de cobertura verano & 0,84 & 0,35 \\
\hline Porcentaje de cobertura otoño & 0,87 & 0,01 \\
\hline Porcentaje de cobertura invierno & 0,64 & 0,12 \\
\hline Diámetro base de la planta & 0,83 & 0,03 \\
\hline Porcentaje de hoja & 0,68 & $-0,55$ \\
\hline Largo de lámina verano & 0,45 & 0,01 \\
\hline Largo de lámina otoño & 0,37 & $-0,03$ \\
\hline Largo de lámina invierno & 0,40 & $-0,08$ \\
\hline Materia seca verano & 0,81 & 0,36 \\
\hline Materia seca otoño & 0,89 & $-0,07$ \\
\hline Materia seca invierno & 0,77 & $-0,01$ \\
\hline Producción de semilla & $-0,31$ & 0,72 \\
\hline Valor propio & 11,18 & 1,98 \\
\hline Variación, \% & 75,32 & 13,33 \\
\hline Variación acumulada, \% & 75,32 & 88,65 \\
\hline
\end{tabular}

accesiones de crecimiento más postrado en la época de otoño e invierno. El Grupo 2 mostró un comportamiento intermedio en producción de forraje, mientras que el Grupo 1 poseía las accesiones con una producción intermedia de semilla y forraje. El Grupo 6 se constituyó por una sola accesión, la número 125 , y se caracterizó por tener la mayor producción de semilla $\left(67,3\right.$ gp planta $\left.^{-1}\right)$ y por ser una planta alta en verano $(35,0 \mathrm{~cm})$.
El resultado de este estudio mostró que para la evaluación de una gran cantidad de accesiones la técnica K-media es una estrategia adecuada para agrupar. Permite formar grupos representativos de recursos genéticos; así por ejemplo las accesiones con alto rendimiento de forraje y alto porcentaje de hoja, estarán incluidas en el Grupo 3. Accesiones con alta producción de semilla, estarán incluidas en el Grupo 4. Para obtener materiales intermedios en ambos 
atributos se incluirá accesiones de los Grupos 2; 1 y 4 . Hasta tener mayor información, tal como a través de marcadores genéticos, estos resultados preliminares sugieren que una estratificación al azar de cuatro accesiones por grupo sería una adecuada representación de la variación fenotípica en esta colección de B. valdivianus.

\section{Análisis discriminante}

Con las pruebas estadísticas de Wilks'Lambda, con un valor de $0,0083(\mathrm{p}<0,01)$ y Hotelling T2 con 14,84 ( $<<0,01)$, se rechaza la hipótesis de igualdad de grupos. Adicionalmente las distancias entre grupos de Malahanobis, indican que los cinco grupos son estadísticamente diferentes. Con este análisis se calcularon nuevas funciones discriminantes (FD), los valores propios respectivos, así como la correlación entre las variables originales con las dos primeras funciones discriminantes (Cuadro 5). La primera función discriminante explicó el $75 \%$ de la variabilidad total. Esta se compuso por factores de producción forrajera: materia seca, cobertura, diámetro, porcentaje de hoja, rebrote, hábito de crecimiento y adaptación. De estos los mayores valores correspondieron a la producción de materia seca en otoño, porcentaje de cobertura en otoño y verano, diámetro base de la planta y materia seca en verano. Todas estas variables se asocian positivamente a esta función. Dos variables presentaron una alta asociación negativa: hábito de crecimiento en otoño e invierno. Esto indicó que las accesiones con una buena aptitud forrajera, presentaron un hábito de crecimiento más erecto en otoño e invierno.

La segunda función discriminante explicó el $13 \%$ de la variabilidad total. Las variables más asociadas a ella se relacionaron a la etapa reproductiva: producción de semilla, número de inflorescencias y altura de planta en verano. El hábito de crecimiento en verano indicó que a medida que las variables ligadas a la producción de semilla aumentaron, las plantas tuvieron un crecimiento más erecto.

Al ordenar las accesiones, basándose en los valores respectivos de las funciones discriminantes, se permite apreciar en forma más objetiva, qué accesiones se destacan en una determinada función. La primera función discriminante explicó el $75 \%$ de la variabilidad total, y se asoció a la producción forrajera. En el Grupo 3 se encuentran las accesiones con mayores rendimientos de materia seca. Destacándose la accesión 120 con un rendimiento 66,0; 64,8 y 22,9 gp planta ${ }^{-1}$; accesión 98 con 86,$3 ; 64,2$ y 24,5 gp planta-1 $^{-1}$ y la accesión 25 con una producción promedio de 62,6; 61,0 y 22,8 gp planta $^{-1}$, en todos los casos en verano, otoño e invierno respectivamente. En cambio, en el Grupo 5 se agruparon las accesiones de menor producción de materia seca: la accesión 109 con rendimientos de 13,$4 ; 12,3$ y 8,6 gp planta $^{-1}$; la accesión 15 con 18,$9 ; 16,4$ y 8,2 gp planta ${ }^{-1}$ y la accesión 35 con 29,1; 16,9 y 8,8 gp planta $^{-1}$, para verano, otoño e invierno respectivamente.

La segunda función discriminante explicó el $13 \%$ de la variación total y se asoció a caracteres relacionados con producción de semilla, destacándose el Grupo 4.

Los cinco grupos (Figura 3) mostraron una significativa distancia entre ellos, especialmente entre los Grupos 3 y 5. La variabilidad dentro de grupos, reflejó la amplia variabilidad fenotípica de esta especie dentro de la Región. Las accesiones de mayor producción de forraje se localizaron hacia la derecha de la Figura 3 (Grupo 3).

Con la aplicación de este análisis se discriminó el germoplasma de $B$. valdivianus, por dos características agronómicas relevantes: la producción de forraje y de semilla. En la literatura no existen referencias de estudios similares con B. valdivianus, pero se reportan trabajos utilizando este análisis. Estos estudios concluyen que es una herramienta muy valiosa en la discriminación de un determinado material en base a la medición de diferentes caracteres. Permite contar con una población de accesiones agrupadas en base a dos atributos fundamentales, como son la producción de forraje y semilla. Además, corrobora lo encontrado previamente, a través de los análisis de componente principal (Figuras 3).

Tradicionalmente, la estimación y clasificación de la distancia genética está basada enteramente en marcadores morfológicos y variables cuantitativas. Los estudios más recientes utilizan marcadores genéticos, como por ejemplo, isoenzimas y marcadores de ADN (Demey, et al., 2003). Sin embargo, estos últimos análisis 


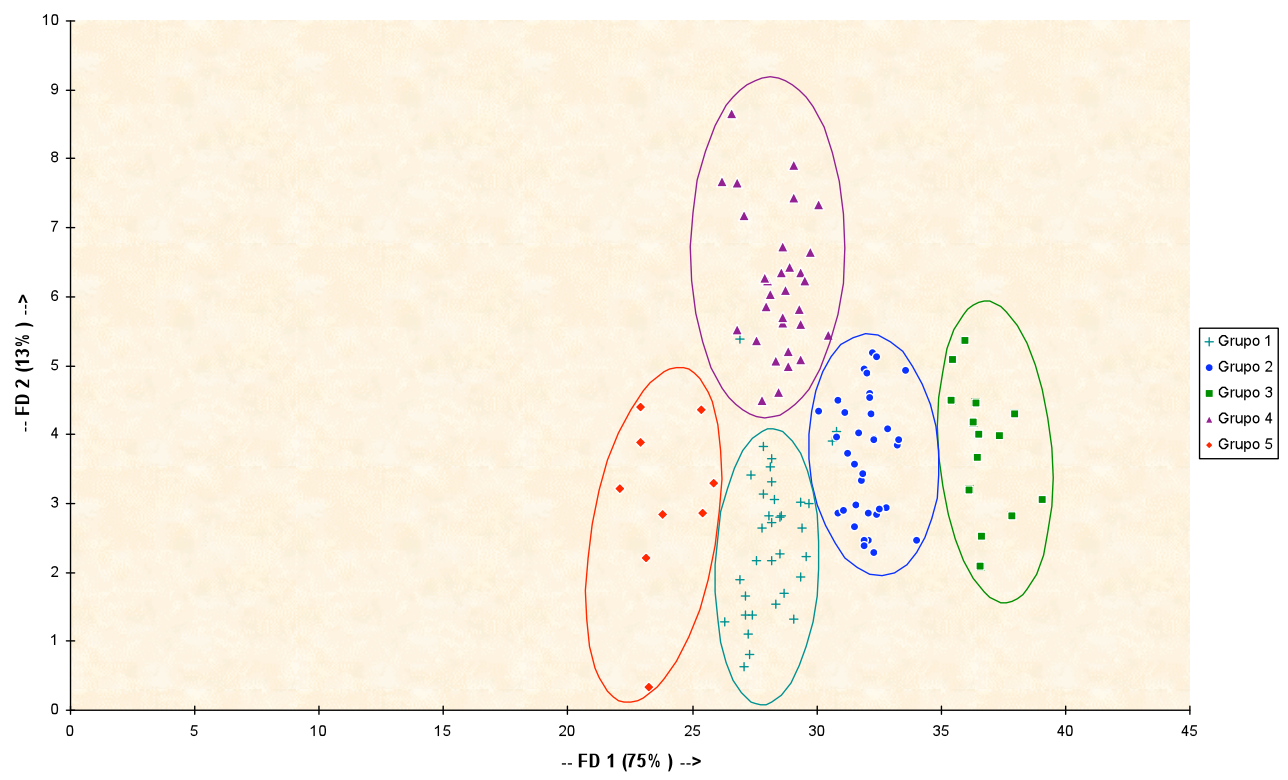

Figura 3. Proyección de los 125 ecotipos de Bromus valdivianus en base a las dos primeras funciones discriminantes

Figure 3. Projection of the 125 ecotypes based on the first two discriminatory functions

están, muchas veces, fuera del alcance de los mejoradores, por sus altos costos, dada la gran cantidad de material que hay que manejar. Por tanto, el tipo de análisis efectuado en este trabajo es una solución práctica para medir la diversidad genética a través de caracteres fenotípicos.

Las accesiones evaluadas de B.valdivianus mostraron una considerable variabilidad fenotípica, principalmente en los caracteres de interés forrajero. Se distinguieron dos grupos de accesiones claramente diferenciadas entre sí, uno constituido por plantas con buena producción de forraje y otro con alta producción de semilla, existiendo grupos con características intermedias.

Los métodos de análisis multivariados utilizados proporcionan un complemento útil para el análisis de datos tradicionales, como los morfológicos y agronómicos. Estos caracteres fenotípicos ayudan en la determinación de la diversidad genética y constituyen una base para un posterior uso en programas de mejoramiento.

\section{AGRADECIMIENTOS}

El presente trabajo fue financiado por la Comisión de Investigación Científica y Tecnológica (Conicyt), proyecto Fondecyt 1951099. Los autores agradecen especialmente la contribución en el área de genética y estadística a los profesores Patricio Barriga (QEPD), de la Universidad Austral de Chile y Michael Casler de la Universidad de Wisconsin, USA.

\section{BIBLIOGRAFIA}

BALOCCHI, O.; LÓPEZ, I. 2001. Rol de las especies pratenses nativas y naturalizadas en las praderas permanentes del Sur de Chile. In: García Cretton, (eds.). Proceedings. Simposio Internacional en Producción Animal y Medio Ambiente. Pontificia Universidad Católica de Chile, Santiago, Chile. pp 285-299 
COMASTRI, J.; POTT, A 1982. Metodología para avaliacao de forrageiras. EMBRAPA-UEPAE Documentos No 2. Corumba, Brasil. 27 p.

DEMEY, J.; ZAMBRANO, A.; FUENMAYOR, F.; SEGOVIA, V. 2003. Relación entre caracterizaciones molecular y morfológica en una colección de yuca. Interciencia 28:684-689.

DERKSEN, D.; LAFOND, G.; THOMAS, A.; LOEPPKY, H.; SWANTON, C.1993. Impact of agronomic practices on weed communities: Tillage systems. Weed Science 41:409-417.

FRANCO, J.; CROSSA, J.; VILLASEÑOR, J.; TABA, S.; EBERHART, S. 1997. Classifying Mexican maize accessions using hierarchical and density search methods. Crop Sci. 37:972-980.

HAIR, J.; ANDERSON, R.; TATHAM, R.; BLACK, W.. 1992. Multivariate data analysis: With readings. NY,USA, Macmillan. 544 p.

HÄRDLE, W.; SIMAR, L.. 2007. Applied multivariate statistical analysis. Second extended ed., Germany, Springer Verlag.456 p.

HAZEN, S.P.; NG, P.K.W.; WARD, R.W. 1997. Variation in grain functional quality for soft winter wheat Crop Sci. 37:1086-1093.

LÓPEZ, I.F.; BALOCCHI, O.; LAILHACAR, P.; OYARZÚN, C.. 1997. Caracterización de sitios de crecimiento de seis especies pratenses nativas y naturalizadas del dominio húmedo de Chile. Agro Sur 25:62-80.

MONTALDO, P.; FUENTES, R.. 1981. Zonificación agroecológica de la Décima Región de Chile II zonas agroclimáticas. Agro Sur 9:70-75.

MONTALDO, P.; Mac DONALD, R.; FUENTES, R 1982. Zonificación agroecológica de la Décima
Región de Chile III zonas agroedáficas. Agro Sur 10:145-151.

MUÑOZ, M. 1980. Flora del Parque Nacional Puyehue. Santiago, Chile, Universitaria. 557 p.

PILLAY, M. 1995. Chloroplast DNA similarity of smooth bromegrass with other pooid cereals: Implications for plant breeding. Crop Sci. 35:869-875.

SMITH, S.; GUARINO, L.; AL-DOSS,; CONTA, D. 1995. Morphological and agronomic affinities among middle eastern alfalfas-accesions from Oman and Yemen. Crop Sci. 35:1180-1194.

STEEL, R.;. TORRIE, J. 1988. Bioestadística: Principios y procedimientos. Segunda ed. Mexico, McGraw-Hill.. 622 p.

STEWART, A. 1996. Potential value of some Bromus species of the section Ceratochloa. New Zealand Journal of Agricultural Research 39:611-618.

SUTHERLAND, B.L: 1994. Dryland pasture and animal evaluation of Grassland Gala grazing brome. Proc. N.Z. Grass. Association 51-81

TOLEDO, J.; SCHULTZE-KRAFT, R. 1982. Metodología para la evaluación agronómica de pastos tropicales In: J. Toledo, J. (ed.). Manual para la Evaluación Agronómica, Red Internacional de Evaluación de Pastos Tropicales. CIAT. Cali, Colombia.pp 91-110

TYLER, B.; HAYES,J.J.; ELLIS-DAVIES, W.. 1985. Forage grass Descriptors. IBGR. Roma. 30 p. VANHALA, T.K.; . VAN RIJN, C.P.E.; BUNTJER, J.; STAM, P.; NEVO, E.; POORTER, H.; VAN EEUWIJK, F.A.. 2004. Environmental, phenotypic and genetic variation of wild barley (Hordeum spontaneum) from Israel. Euphytica 137:297-309. 